\title{
MicroRNA-134 suppresses cell proliferation in gastric cancer cells via targeting of GOLPH3
}

\author{
YANQI LIU ${ }^{1 *}$, YANHONG SUN ${ }^{2 *}$ and AIZHEN ZHAO ${ }^{3}$ \\ ${ }^{1}$ Department of Gastroenterology, The Affiliated Hospital of Inner Mongolia Medical University; \\ ${ }^{2}$ Department of Physiology, Inner Mongolia Medical University; ${ }^{3}$ Department of Ultrasound, \\ The Affiliated Hospital of Inner Mongolia Medical University, Hohhot, Inner Mongolia 010050, P.R. China
}

Received August 13, 2016; Accepted November 25, 2016

DOI: $10.3892 / o r .2017 .5488$

\begin{abstract}
Emerging evidence has shown that microRNAs (miRNAs) play critical roles in tumor development and progression. miR-134 has been found to act as a tumorsuppressor in numerous types of cancers. However, little is known concerning the potential role of miR-134 in gastric carcinogenesis. In the present study, we found that miR-134 was highly downregulated in gastric cancer tissues and cell lines when compared with levels in their adjacent non-tumor tissues and the normal human gastric epithelial cell line GES-1. Additionally, overexpression of miR-134 was accompanied by reduced cell proliferation in vitro and decreased tumor size in vivo. Further investigation by luciferase reporter assay indicated that Golgi phosphoprotein 3 (GOLPH3), a potent oncogene, was a direct target of miR-134. The activity of a luciferase reporter carrying the miR-134 binding site in the 3'-untranslated region (3'-UTR) of GOLPH3 was repressed by overexpression of miR-134, while a mutation in the 3 '-UTR of GOLPH3 abrogated this effect, indicating that GOLPH3 is a target gene of miR-134. Overexpression of GOLPH3 blocked the antiproliferative effect of pre-miR-134 in gastric carcinoma cells. Furthermore, overexpression of miR-134 was associated with decreased phosphorylation of AKT, mTOR and S6K. Taken together, these data suggest that miR-134 regulates gastric cancer cell proliferation, at least potentially, through downregulation of the GOLPH3 gene, implicating a candidate tumor-suppressor miRNA in the pathogenesis of gastric cancer.
\end{abstract}

Correspondence to: Dr Aizhen Zhao, Department of Ultrasound, The Affiliated Hospital of Inner Mongolia Medical University, 1 Tongdao North Road, Hohhot, Inner Mongolia 010050, P.R. China E-mail: aizhenzhaoazz@163.com

*Contributed equally

Key words: gastric cancer, proliferation, microRNA-134, GOLPH3, pathogenesis

\section{Introduction}

Gastric cancer (GC) ranks as the fifth most common cancer and is the third leading cause of cancer-related death worldwide (1). Despite a steadily declining incidence, GC remains a highly fatal disease that causes more than 723,000 deaths per year (2). In addition, due to its silent nature and underlying genetic and biological heterogeneity, the prognosis of GS has improved little over time (1). Therefore, there is an urgent need to improve GC patient outcomes by achieving a detailed understanding of the molecular mechanism underlying GC.

MicroRNAs (miRNAs) are a class of $\sim 22$ nucleotides long, endogenous, small, non-coding RNAs that negatively regulate genes by triggering either mRNA degradation or translational repression through base pairing to the $3^{\prime}$-untranslated region (3'-UTR) of specific target mRNAs $(3,4)$. Compelling evidence indicates that more than 200 miRNAs are involved and functional in GC pathogenesis and treatment response $(5,6)$. For example, oncogenic miR-21 and miR-130b were reported to be upregulated in GC and to negatively target the tumor suppressor genes PTEN and RUNX3 $(7,8)$. Reciprocally, tumor-suppressor miRNAs, such as miR-101 and miR-375, were found to be downregulated in GC and led to overexpression of the oncogenic genes EZH2 and PDK1 $(9,10)$. Apart from these miRNAs, miR-134 has been revealed to be downregulated in many solid cancers (11-13). In gastrointestinal stromal tumors, miR-134 was confirmed to be downregulated (14), suggesting a conceivable role of miR-134 in the pathogenesis of GC. However, the detail molecular mechanism needs to be elucidated.

Golgi phosphoprotein 3 (GOLPH3), also known as GPP34 and GMx33, is a 34-kDa Golgi-localizing protein that was originally identified in the mouse Golgi apparatus by Snyder et al (15). GOLPH3 was identified as a novel oncogene in several solid tumors, including lung, prostate, breast, ovarian and pancreatic cancers, and melanomas (16). In patients with GC, GOLPH3 has been reported to be upregulated and is associated with poor clinical outcomes (17). These studies imply that GOLPH3 is a promising molecular target for the prevention of GC.

In the present study, using both in vitro and in vivo models, we investigated the role of miR-134 in regulating GC cell lines and tissues, and the underlying mechanism. miR-134 
was found to markedly inhibit GC cell proliferation and target GOLPH3 via its 3'-UTR region. Moreover, miR-134 expression was inversely correlated with GOLPH3 protein level in GC. Overexpression of GOLPH3 was able to partially reverse the inhibition of GC cell proliferation caused by miR-134. Thereby, these data suggest that miR-134 functions as a tumor suppressor by negatively regulating the GOLPH3 oncogene, which promotes GC proliferation.

\section{Materials and methods}

Patient samples. Twenty-six human GC specimens and pair-matched gastric adjacent non-tumor tissues were obtained from patients undergoing surgical resection at the Department of Gastroenterology, The Affiliated Hospital of Inner Mongolia Medical University (Hohhot, China). GC diagnosis was confirmed by pathologic examination. Informed consent was obtained from all patients before collection. The use of human tissue samples was approved by the Ethics Committee of the Inner Mongolia Medical University.

Cell culture. Human GC cell lines AGS, SNU-1, BGC-823, MGC-803 and SGC-7901 were purchased from the American Type Culture Collection (ATCC; Manassas, VA, USA). Immortalized normal human gastric epithelial cell line GES-1 was obtained from the Institute of Biochemistry and Cell Biology at the Chinese Academy of Sciences (Shanghai, China). The entire cell lines were maintained in RPMI-1640 medium (Gibco, Rockville, MD, USA) supplemented with $10 \%$ fetal bovine serum (FBS) containing $1 \%$ penicillin/ streptomycin in a humidified atmosphere containing $5 \% \mathrm{CO}_{2}$ at $37^{\circ} \mathrm{C}$.

Xenograft tumor assay. Four-week-old female BALB/c athymic nude mice weighing 15-20 g were purchased from the Experimental Animal Centre of the Inner Mongolia Medical University, and raised in a specific pathogen-free environment. Mice were inoculated subcutaneously with a $100-\mu 1$ injection of $1 \times 10^{6}$ BGC- 823 cells transfected either with the miR134-overexpressing or the control vector into the right flanks of the mice. Tumor dimensions were measured every 2 days and tumor volume was calculated according to the following formula: Volume $=$ tumor length $\mathrm{x}$ tumor $\mathrm{width}^{2} / 2$. At day 50 , all mice were sacrificed and tumors were weighed. All animal care and treatments were performed in strict accordance with the National Institutes of Health Guide for the Care and Use of Laboratory Animals and were approved by the Institutional Animal Care Committee of the Inner Mongolia Medical University.

Real-time PCR analysis. Total RNA from sample tissues and cell lines were extracted using TRIzol (Invitrogen, Carlsbad, CA, USA) according to the manufacturer's instructions. Then, reverse transcription reactions were carried out using the One Step PrimeScript miRNA cDNA Synthesis kit and PrimeScript RT-PCR kit (both from Takara, Dalian, China) for miRNA and mRNA analysis, respectively. RT-qPCR analysis was conducted utilizing SYBR-Green qPCR Master Mix (Thermo Fisher, Shanghai, China) and 7900HT Fast Real-Time PCR System (Applied Biosystems, Darmstadt, Germany). The primers used were as follows: 5'-GGTGTGACTGGTTGACCA-3' (forward) and 5'-TGCGTGTCGTGGAGTC-3' (reverse) for miR-134; and 5'-TGTAAGTCAGATGCTCCAACAGG-3' (forward) and 5'-TCACCCATTTGTCAGAACGG-3' (reverse) for GOLPH3. Relative expression of the target genes was normalized to U6 snRNA (for miRNA) or GAPDH (for mRNA) using the $2^{-\Delta \Delta \mathrm{Ct}}$ method.

Cell proliferation assay. To investigate the effect of miR-134 on the proliferation of GC cell lines, $2 \times 10^{4}$ cells were transfected with different concentrations $(0,20,40$ or $80 \mathrm{nM})$ of pre-miR-134 (miR-134 precursors) or pre-miRNA negative control (negative control) an placed into a 96-well plate. After incubation for $24,48,72$ and $96 \mathrm{~h}, 20 \mu \mathrm{l}$ of MTT $(5 \mathrm{mg} / \mathrm{ml})$ was added to each well and the mixture was cultured for another $4 \mathrm{~h}$. Afterwards, $200 \mu \mathrm{l} /$ well of dimethyl sulfoxide was added to dissolve the formazan crystals. Finally, the absorbance at $490 \mathrm{~nm}$ was measured using an ELISA reader (Bio-Tek Instruments Inc., Winooski, VT, USA).

Western blot analysis. Cells were lysed with RIPA buffer (Sigma-Aldrich, St. Louis, MO, USA). Protein concentration was assayed using the Bradford method and a total of $30 \mu \mathrm{g}$ protein was separated through $12 \%$ SDS-PAGE gels. Then, proteins were electrophoretically transferred to a nitrocellulose membrane (Bio-Rad, Hercules, CA, USA). After blocking with $3 \%$ non-fat milk in TBS for $1 \mathrm{~h}$ at $37^{\circ} \mathrm{C}$, the membranes were incubated with primary antibodies at $4^{\circ} \mathrm{C}$ overnight. HRP-conjugated secondary antibodies were then added and incubated for $1 \mathrm{~h}$ at room temperature. Finally, protein bands were visualized using an enhanced chemiluminescence kit (Amersham, Little Chalfont, UK). Anti-human antibodies used in western blot analysis were as follows: GOLPH3 (1:1,000; Abcam, Cambridge, UK), AKT (1:1,500), p-AKT $(1: 1,500)$, mTOR (1:1,000), p-mTOR (Ser2448, 1:1,000), S6K (1:1,500), p-S6K (1:1,500), and HRP-conjugated secondary antibody $(1: 2,000)$ (both from Santa Cruz Biotechnology, Santa Cruz, CA, USA.

Luciferase reporter assay. To validate the target gene of miR-134, a luciferase reporter assay was performed using the pGL3 luciferase promoter vector (Promega, Madison, WI, USA). The cDNA fragments of the human GOLPH3 3'-UTR containing the putative miR-134 binding sites were amplified and subcloned into the luciferase reporter vector pGL3 between the $X b a \mathrm{I}$ and $F s e \mathrm{I}$ restriction sites. As a control, the pGL3-mut-GOLPH3 plasmids were constructed using the 3'-UTR with the miR-134 binding sites mutated. Subsequently, $1 \times 10^{5}$ HEK-293T cells were plated on 24 -well plates, and transfected with $80 \mathrm{ng}$ of pGL3-GOLPH3 or pGL3-mut-GOLPH3 vectors in the presence of $40 \mathrm{nM}$ pre-miR-134 or pre-miR negative controls using Lipofectamine 2000 (Invitrogen). After transfection for $24 \mathrm{~h}$, cells were harvested and the luciferase activities were measured using the Dual-Luciferase Reporter Assay kit (Promega).

Statistical analysis. Statistical analysis was conducted using the Student's t-test. Multiple comparisons were analyzed by one-way analysis of variance. Results were considered statistically significant at $\mathrm{P}<0.05$. 
A

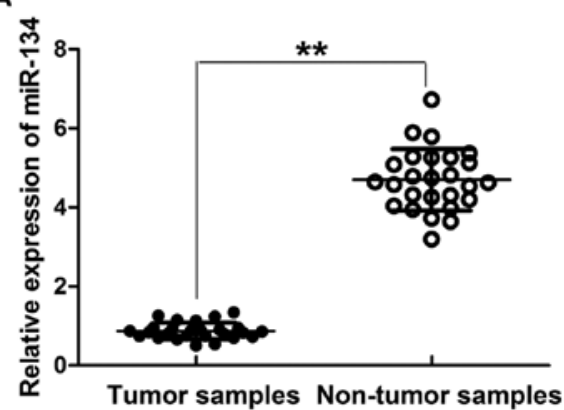

B

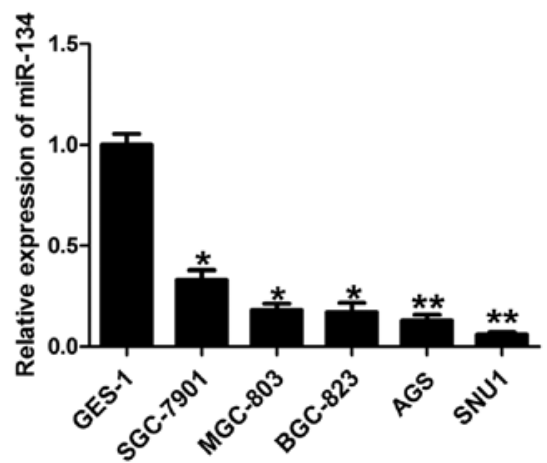

Figure 1. Expression of miR-134 is downregulated in GC. (A) RT-qPCR results indicated a lower level of relative miR-134 expression in 26 primary GC tissues compared with the respective adjacent non-tumor tissues; ${ }^{* *} \mathrm{P}<0.01$. (B) Relative expression of miR-134 in GES-1 and 6 GC cell lines with different differentiation statuses; ${ }^{\mathrm{P}}<0.05,{ }^{* *} \mathrm{P}<0.01$ vs. GES-1 cells.

\section{Results}

miR-134 is downregulated in GC. Previous research has identified consistently decreased miR-134 expression in many types of cancers (11-13). To investigate miR-134 expression in GC, we collected 26 human GC specimens and pair-matched gastric adjacent non-neoplastic mucosa. Compared with adjacent non-tumor tissues, the expression of mature miR-134 was significantly downregulated in the GC tissues (Fig. 1A). Similarly, we observed frequent downregulation of miR-134 in different GC cell lines compared with the immortalized gastric epithelial cell line GES-1 with no tumorigenic effect (Fig. 1B). Moreover, the expression level of miR-134 was positively correlated with the differentiation degree of GC cells by virtue of the lowest expression of miR-134 being observed in the most aggressive cancer cell lines, AGS and SNU1, and the highest expression being found in the GES-1 line. These data suggest a negative correlation between miR-134 expression and GC.

Overexpression of miR-134 suppresses GC cell growth. To fully dissect the potential role of miR-134 in gastric carcinogenesis, we examined the biological function of miR-134 in AGS and BGC-823, two commonly used gastric adenocarcinoma cell lines. GC cells were transfected with either miR-134 precursor (pre-miR-134) or negative control precursor (pre-miR-NC) at different concentrations $(0,20,40$ and $80 \mathrm{nM})$ and incubated for 24,48 and $72 \mathrm{~h}$, respectively. The transfection efficiencies of miR-134 were detected by RT-qPCR (Fig. 2A and B). As shown in Fig. $2 \mathrm{C}$ and $\mathrm{D}$, cell proliferation was significantly suppressed by miR-134 overexpression in both AGS and BGC-823 cells in a dose-dependent manner. In addition, a longer duration of miR-134 transfection markedly attenuated AGS (Fig. 2E) and BGC-823 cell (Fig. 2F) proliferation. These data suggest that miR-134 may be important for gastric carcinogenesis.

Overexpression of miR-134 suppresses xenograft tumor growth in vivo. To verify the positive role of miR-134 in gastric tumor progression in vivo, we engineered the miR-134 overexpression nude mouse model by subcutaneously inoculating AGS cells with miR-134 into nude mice (AGS with miR-NC was used as a control). Not surprisingly, overexpression of miR-134 revealed a significant decrease in tumor volume when compared with the control group (Fig. 3A). Additionally, forced expression of miR-134 resulted in markedly lower tumor weight than the control group (Fig. 3B). Altogether, these results demonstrated that miR-134 is essential for controlling gastric tumor formation.

miR-134 targets the 3'-UTR of GOLPH3. To delineate the underlying molecular mechanism by which miR-134 inhibits GC, we performed bioinformatic analysis to screen the predicted target genes of miR-134. Notably, we discovered that in both TargetScan and PicTar databases, GOLPH3 was a possible predicted target with conserved complementary 'seed' sites for miR-134 in the 3'-UTR in many species, including hsa, rno, mmu and cfa (Fig. 4A). Moreover, a recent study showed that the mRNA level of GOLPH3 is upregulated in GC tissue and is positively associated with cell proliferation (18). Given this evidence and the observed function in miR-134-overexpressing cell lines and mice, we speculated that GOLPH3 was a target of miR-134. To test this hypothesis, we co-transfected pGL3-GOLPH3 or pGL3-mut-GOLPH3 vectors in the presence of pre-miR-134 or pre-miR-NC into HEK293T cells. The result of the luciferase reporter assay showed that HEK293T cells transfected with the 3'-UTR of GOLPH3 and pre-miR134 exhibited decreased luciferase activity compared with the pre-miR-NC, while mutation at the 3'-UTR of GOLPH3 lost this response (Fig. 4B).

Given that microRNAs regulate genes by triggering either mRNA degradation or translational repression, we also tested the expression of GOLPH3 mRNA and protein levels in AGS and the BGC-823 cells. An $\sim 63$ and $74 \%$ reduction in GOLPH3 protein expression was found in the miR-134-overexpressing AGS and BGC-823 cell lines, respectively (Fig. 4C). However, overexpression of miR-134 had no effect on GOLPH3 mRNA levels (Fig. 4D). Moreover, compared with the adjacent non-tumor tissues, the expression of GOLPH3 was markedly upregulated in GC tissues (Fig. 4E).

The cell growth-inhibiting activity of miR-134 is abrogated by GOLPH3. To further validate the hypothesis that miR-134 regulates the growth of GC cells through GOLPH3, we co-transfected pre-miR-134 and GOLPH3 overexpression vectors harboring no specific miR-134-binding sequences in the 3'-UTR in AGS and BGC-823 cells. Co-transfection 




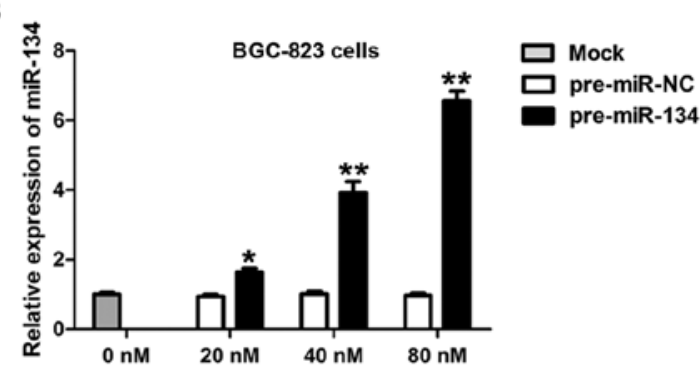

C

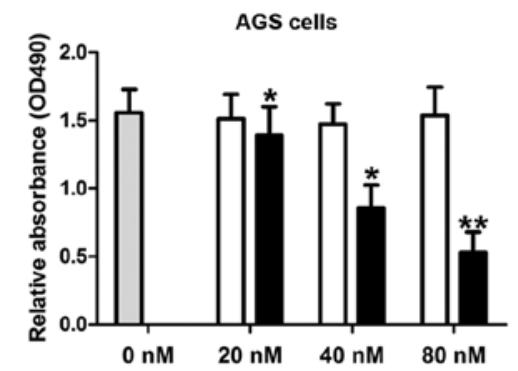

E

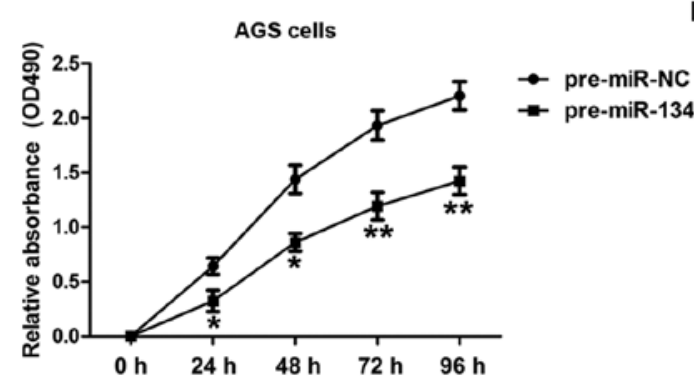

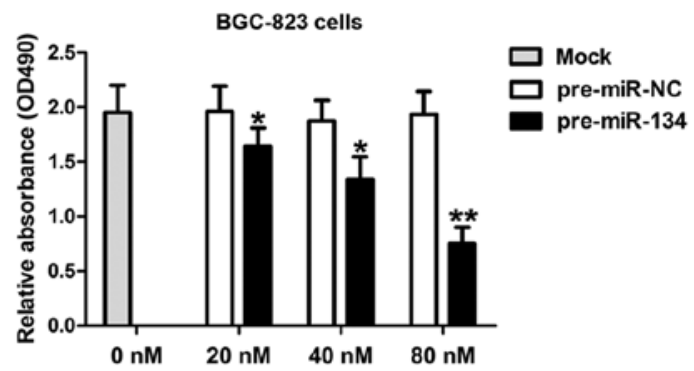

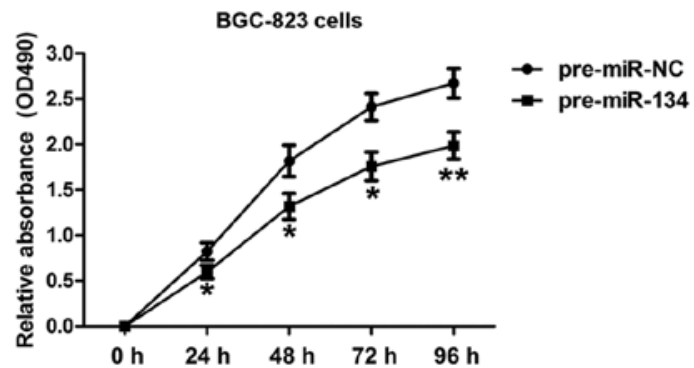

Figure 2. miR-134 suppresses GC cell proliferation. AGS and BGC-823 cells were transfected with miR-134 precursor (pre-miR-134) or negative control precursor (miR-NC) at different concentrations $(0,20,40$ and $80 \mathrm{nM})$ and incubated for $0,24,48,72$ and $96 \mathrm{~h}$, respectively. The transfection efficiencies of miR-134 were detected by RT-qPCR in (A) AGS and (B) BGC-823 cells. MTT assay showed that miR-134 overexpression reduced the proliferation rate of the (C) AGS and (D) BGC-823 at different concentrations. (E and F) Cells were treated with $40 \mathrm{nM}$ pre-miR-134 or pre-miR-NC, and the proliferation rate was analyzed by MTT assay at different times; ${ }^{\mathrm{P}} \mathrm{P}<0.05,{ }^{* *} \mathrm{P}<0.01$ vs. the pre-miR-NC.

A

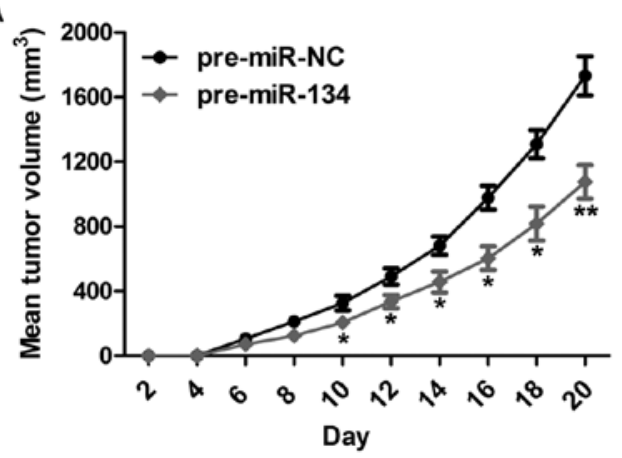

B

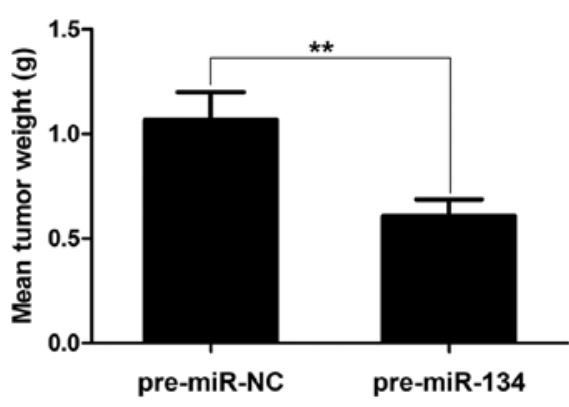

Figure 3. Overexpression of miR-134 suppresses xenograft tumor growth in vivo. Nude mice were subcutaneously inoculated with AGS cells with miR-134 overexpression. miR-134 overexpression resulted in suppressed (A) xenograft tumor volume and (B) tumor weight in vivo; ${ }^{*} \mathrm{P}<0.05,{ }^{* *} \mathrm{P}<0.01 \mathrm{vs}$. the pre-miR-NC.

of miR-134 and GOLPH3 induced an increased expression of GOLPH3 when compared with pre-miR-134 transfection alone in both AGS (Fig. 5A) and BGC-823 cells (Fig. 5B). Furthermore, cell proliferation was evaluated $24 \mathrm{~h}$ after transfection using the MTT assay. As shown in Fig. 5C and D, transfection with pre-miR-134 inhibited cell proliferation compared with the control, while increasing GOLPH3 protein expression effectively reversed the cell growth arrest induced by miR-134 overexpression in both AGS and BGC-823 cells.

Expression of miR-134 inversely correlates with the activation of mTOR. GOLPH3 is a Golgi-localizing protein playing an important role in Golgi to plasma membrane trafficking (19). It has been previously reported that GOLPH3 contributes to 


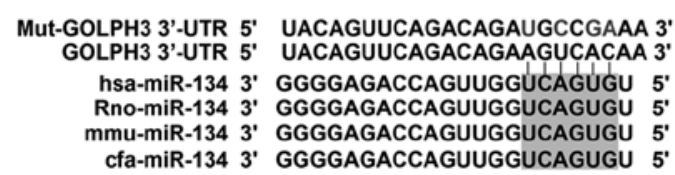

C
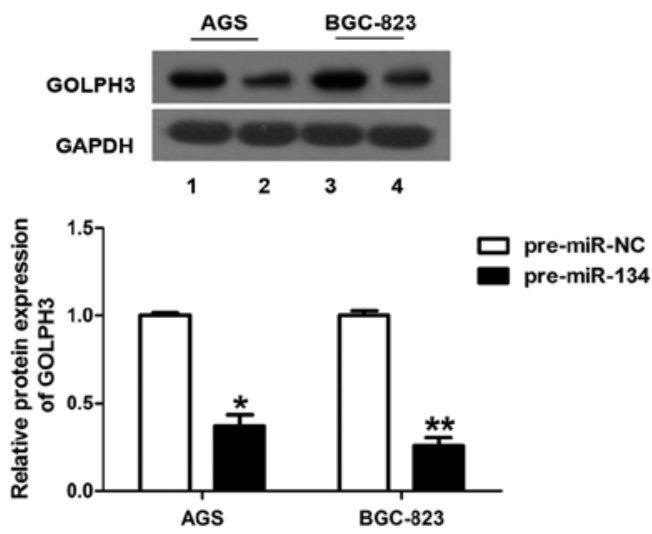

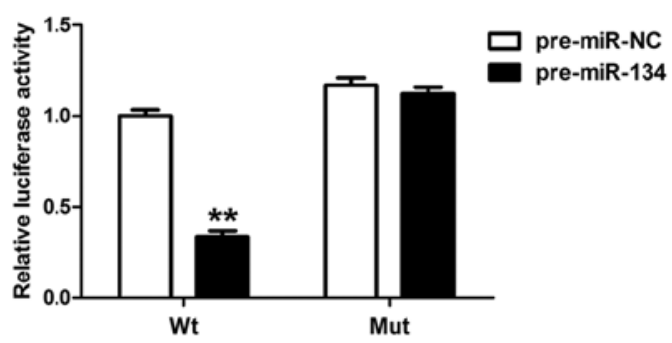

D

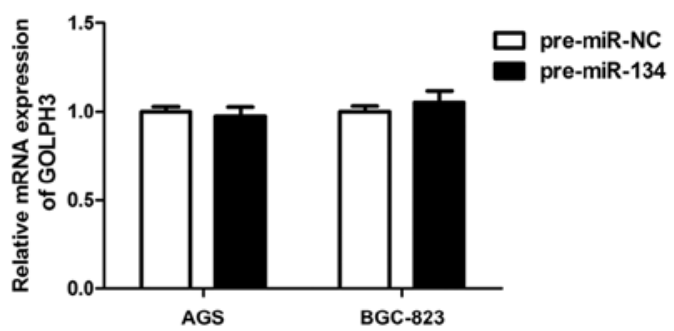

E

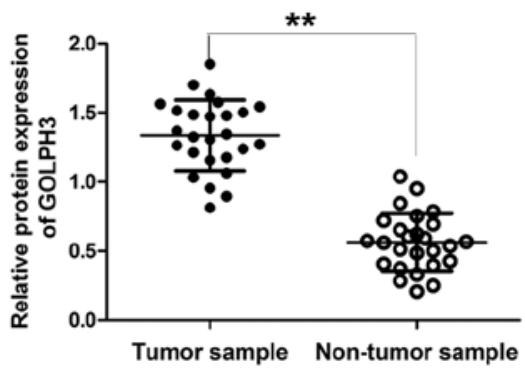

Figure 4. GOLPH3 is a direct target of miR-134. (A) Alignment of miR-134 with GOLPH3 3'-UTR sequences. (B) HEK293T cells were co-transfected with luciferase reporter with wild-type (WT) GOLPH3 3'-UTR or with mutant GOLPH3 3'-UTR, and pre-miR-134 or negative control precursor (pre-miR-NC) for $48 \mathrm{~h}$. The relative luciferase activity was analyzed by luciferase assay. (C) Western blot results showed that overexpression of miR-134 markedly decreased GOLPH3 protein levels in AGS and BGC-823 cell lines. Lanes 1 and 3 indicate pre-miR-NC; lanes 2 and 4 indicate pre-miR-134. (D) RT-qPCR results of GOLPH3 mRNA levels in miR-134-overexpressing AGS and BGC-823 cell lines. (E) Western blot results showed a higher level of relative GOLPH3 expression in 26 primary GC tissues compared with the respective adjacent non-tumor tissues; ${ }^{*} \mathrm{P}<0.05,{ }^{* *} \mathrm{P}<0.01$ vs. the pre-miR-NC.



$\mathbf{B}$

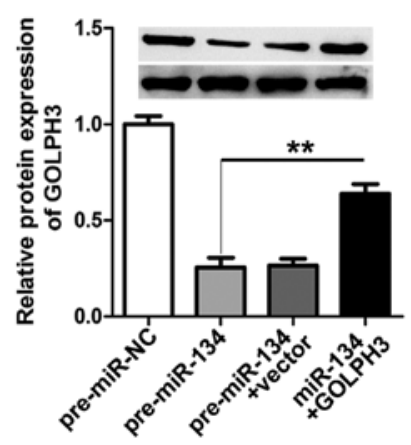

C

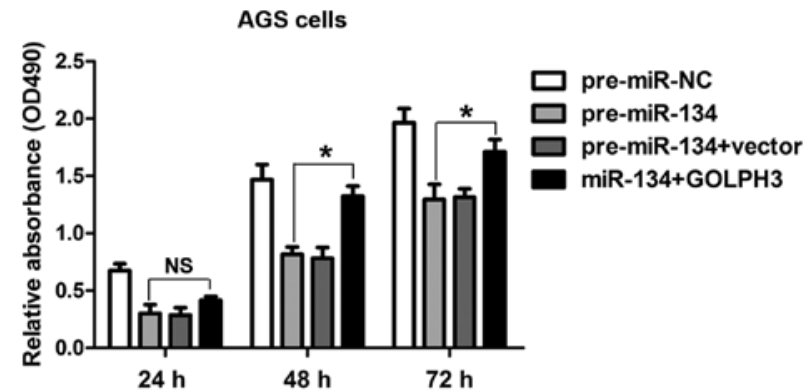

D



Figure 5. GOLPH3 abrogates the inhibitory effect of miR-134 on cell proliferation. Cells were treated with 40 nM pre-miR-134 or pre-miR-134 plus GOLPH3. The protein levels of GOLPH3 were analyzed using western blotting in (A) AGS and (B) BGC-823 cell lines. (C and D) The proliferation rate was determined by MTT assay at different time points; ${ }^{*} \mathrm{P}<0.05,{ }^{* *} \mathrm{P}<0.01 \mathrm{vs}$. the pre-miR-134. 
A

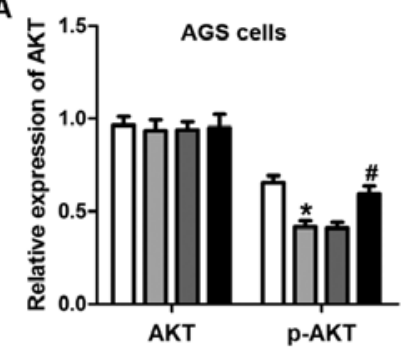

B

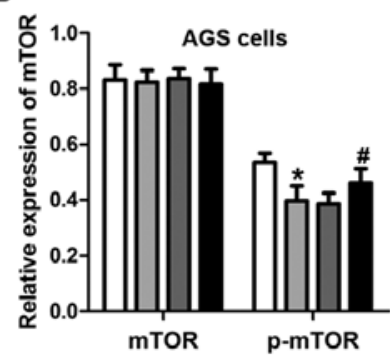

C



D

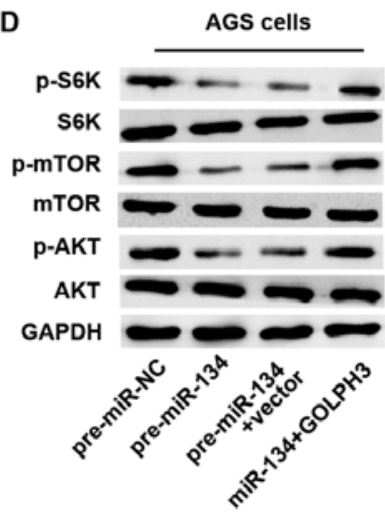

E
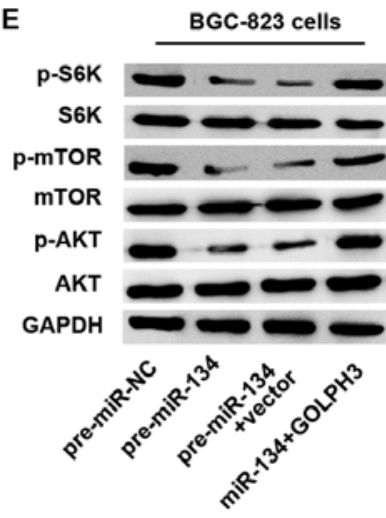

F

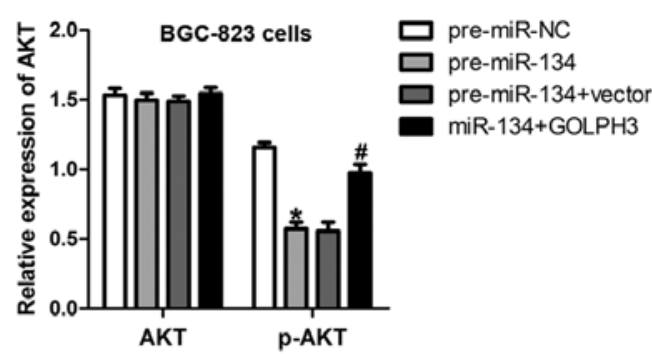

G

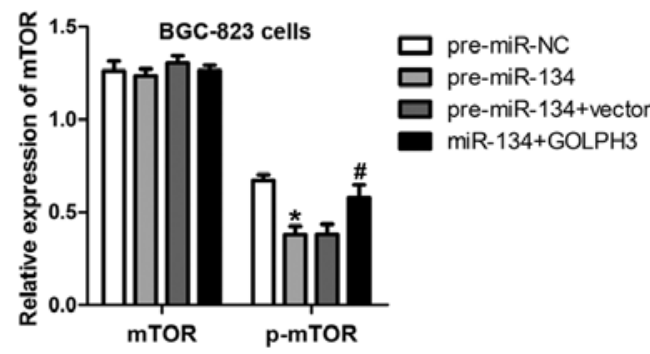

H

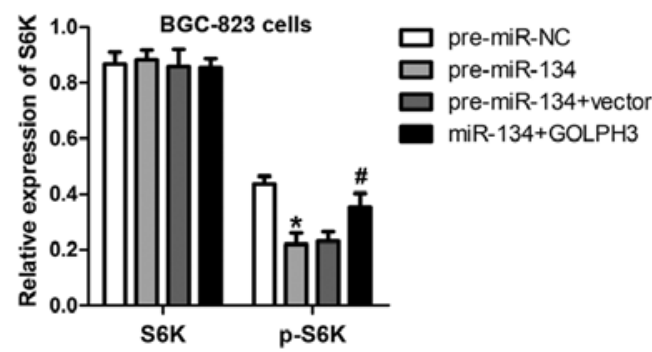

Figure 6. Effects of miR-134 and GOLPH3 on Akt, mTOR and p70s6k expression. Cells were treated with $40 \mathrm{nM}$ pre-miR-134 or pre-miR-134 plus GOLPH3. Relative expression of (A) Akt, (B) mTOR and (C) p70s6k in AGS cells was determined using western blotting. Quantitative analysis of Akt, mTOR and p70s6k expression in (D) AGS and (E) BGC-823 cells. Relative expression of (F) Akt, (G) mTOR and (H) p70s6k in BGC-823 cells was detected using western blotting; "P<0.05 vs. the pre-miR-NC; ${ }^{\#} \mathrm{P}<0.05$ vs. the pre-miR-134.

the activation of mTOR and p70s6k (16), which are involved in cell proliferation and growth (20). Considering the regulatory role of miR-134 in GOLPH3, we speculated that miR-134 decreased mTOR and p70s6k phosphorylation and activation through decreasing GOLPH3. To validate this hypothesis, we examined Akt, mTOR and p70s6k phosphorylation levels after miR-134 overexpression or miR-134-GOLPH3 overexpression using western blot analysis. As predicted, the phosphorylation of Akt, mTOR and p70s6k was apparently inhibited by the overexpression of miR-134 (Fig. 6), while overexpression of GOLPH3 restored this effect caused by miR-134. These data imply that Akt, mTOR and p70s6k signaling were impaired by miR-134 overexpression.

\section{Discussion}

miRNA deregulation has been correlated with the progression and prognosis of GC since the discovery of aberrant miRNA expression patterns in GC patients compared with normal controls $(21,22)$. miRNA microarrays showed that miR-1 and miR-101, whose downregulation was connected with various types of cancers $(9,23)$, were downregulated in gastric tissues from GC patients (24). miR-375 targets the Janus kinase 2 (JAK2) gene in GC patient gastric tissues (25), and may represent a predictor of GC progression (26). Although altera- tions in differentially regulated miRNAs have been identified in GC, the present study provides insight into miR-134 and its target gene in GC, which further defines the mechanisms underlying GC. Previous studies identified a close relationship between miR-134 and the progression of various types of cancers. In patients with colorectal cancer, osteosarcoma and breast cancer, miR-134 was found to be decreased and to act as a tumor-suppressor (11-13). In the present study, we provide evidence that miR-134 is downregulated in GC tissues and cell lines. Gene ontology analysis suggests that miR-134-regulated genes are highly involved in cancer differentiation and proliferation pathways. Potential direct targets of miR-134 that are relevant to cell growth were identified by virtue of their upregulation in gastric cell lines and in mice engineered to overexpress miR-134. Indeed, we identified that overexpression of miR-134 inhibited proliferation in GC cells and tumor formation in xenograft tumor assays, indicating a tumor-suppressor role of miR-134 in GC.

To the best of our knowledge, miRNAs function by regulating their target genes. Using two miRNA target prediction algorithms (PicTar and TargetScan), we predicted $>100$ target genes of miR-134. Among them, GOLPH3 was outstanding for the following reasons. i) The 3'-UTR of GOLPH3 was predicted to be a highly conserved binding site for miR-134 in several species: hsa, rno, mmu and cfa. ii) GOLPH3 is an oncogene 
in many solid tumors (16), and a previous clinical study indicated that GOLPH3 is overexpressed in patients with GC and is associated with poor clinical outcome in GC (17). iii) Apart from GOLPH3, other proteins from the GOLPH family, such as GOLPH2, were identified to be ectopically expressed in GCs (27). iv) Even though a full underlying molecular mechanism was not observed, GOLPH3 was proven to play an essential role in vesicle trafficking and Golgi structure (28). Moreover, Field et al found that overexpression of GOLPH3 confers resistance to killing by DNA-damaging agents and depletion of GOLPH3 could enhance the ability of DNA-damaging agents to kill cells (29). Among potential direct targets of miR-134, GOLPH3 was identified by the presence of a match to the miR-134 seed sequence in the 3'-UTR of GOLPH3 by luciferase assay. The result showed that HEK293T cells transfected with the 3'-UTR of GOLPH3 and pre-miR-134 exhibited decreased luciferase activity compared with the pre-miR-NC, while mutation at the 3'-UTR of GOLPH3 lost this response.

In addition to the gene regulation discussed in the present study, we identified the pathway involved in this process. It has been previously reported that GOLPH3 functions in the mTOR pathway based in cancer $(16,30)$. Similarly, Murayama et al reported that $\mathrm{p}-\mathrm{mTOR}$ expression was positively correlated with GC tumor invasion and tumor stage (31). In addition, a preliminary study revealed that GOLPH3 was positively correlated with Akt/mTOR activation in GC (18). In line with these studies, we showed in the present study that the phosphorylation of AKT, mTOR and mTOR downstream substrate protein S6K were impaired by miR-134 overexpression, while overexpression of GOLPH3 restored this effect caused by miR-134, suggesting that miR-134 regulated this AKT/mTOR/S6K signaling pathway via a GOLPH3-dependent mechanism.

In conclusion, miR-134, which is downregulated in gastric tumor tissues and cell lines, negatively regulates gastric tumor growth and cell proliferation. Furthermore, miR-134 inhibits GC cell proliferation by targeting GOLPH3 and subsequently, the AKT/mTOR/S6K pathway. To the best of our knowledge, this is the first time that miR-134 has been shown to target GOLPH3 in GC cells. Therefore, further research exploring the anticancer role of miR-134 may contribute to the development of new therapeutic strategies for GC.

\section{Acknowledgements}

The present study was supported by the Scientific Research Projects of Inner Mongolia Autonomous Region Colleges and Universities in 2016 (grant no. NJZY16113).

\section{References}

1. Tan P and Yeoh KG: Genetics and molecular pathogenesis of gastric adenocarcinoma. Gastroenterology 149: 1153-1162.e3, 2015.

2. Ferlay J, Soerjomataram I, Ervik M, Dikshit R, Eser S, Mathers C, Rebelo M, Parkin DM, Forman D and Bray F: GLOBOCAN 2012 v1.0, Cancer Incidence and Mortality Worldwide: IARC CancerBase No. 11 (Internet). International Agency for Research on Cancer, Lyon, France, 2013. http:// globocan.iarc.fr.

3. Ambros V: The functions of animal microRNAs. Nature 431: 350-355, 2004.

4. Bartel DP: MicroRNAs: Genomics, biogenesis, mechanism, and function. Cell 116: 281-297, 2004.
5. Song JH and Meltzer SJ: MicroRNAs in pathogenesis, diagnosis, and treatment of gastroesophageal cancers. Gastroenterology 143: 35-47.e2, 2012.

6. Song S and Ajani JA: The role of microRNAs in cancers of the upper gastrointestinal tract. Nat Rev Gastroenterol Hepatol 10: 109-118, 2013.

7. Zhang BG, Li JF, Yu BQ, Zhu ZG, Liu BY and Yan M: microRNA-21 promotes tumor proliferation and invasion in gastric cancer by targeting PTEN. Oncol Rep 27: 1019-1026, 2012.

8. Lai KW, Koh KX, Loh M, Tada K, Subramaniam MM, Lim XY, Vaithilingam A, Salto-Tellez M, Iacopetta B, Ito Y, et al; Singapore Gastric Cancer Consortium: MicroRNA-130b regulates the tumour suppressor RUNX3 in gastric cancer. Eur J Cancer 46: 1456-1463, 2010.

9. Varambally S, Cao Q, Mani RS, Shankar S, Wang X, Ateeq B, Laxman B, Cao X, Jing X, Ramnarayanan K, et al: Genomic loss of microRNA-101 leads to overexpression of histone methyltransferase EZH2 in cancer. Science 322: 1695-1699, 2008.

10. Tsukamoto Y, Nakada C, Noguchi T, Tanigawa M, Nguyen LT, Uchida T, Hijiya N, Matsuura K, Fujioka T, Seto M, et al: MicroRNA-375 is downregulated in gastric carcinomas and regulates cell survival by targeting PDK1 and 14-3-3zeta. Cancer Res 70: 2339-2349, 2010.

11. Xie Y, Song J, Zong Q, Wang A, Yang Y, Liu F and Meng X: Decreased expression of miR-134 and its clinical significance in human colorectal cancer. Hepatogastroenterology 62: 615-619, 2015.

12. Bao Y, Peng L, Ma J, Liu K and Li W: Decreased miR-134 expression and its tumor-suppressive function in human osteosarcoma. Genet Mol Res 14: 16771-16781, 2015.

13. Zhang J, Ma Y, Wang S, Chen F and Gu Y: C/EBP $\alpha$ inhibits proliferation of breast cancer cells via a novel pathway of miR-134/CREB. Int J Clin Exp Pathol 8: 14472-14478, 2015.

14. Haller F, von Heydebreck A, Zhang JD, Gunawan B, Langer C, Ramadori G, Wiemann S and Sahin O: Localization- and mutationdependent microRNA (miRNA) expression signatures in gastrointestinal stromal tumours (GISTs), with a cluster of coexpressed miRNAs located at 14q32.31. J Pathol 220: 71-86, 2010.

15. Snyder CM, Mardones GA, Ladinsky MS and Howell KE: GMx33 associates with the trans-Golgi matrix in a dynamic manner and sorts within tubules exiting the Golgi. Mol Biol Cell 17: 511-524, 2006.

16. Scott KL, Kabbarah O, Liang MC, Ivanova E, Anagnostou V, Wu J, Dhakal S, Wu M, Chen S, Feinberg T, et al: GOLPH3 modulates mTOR signalling and rapamycin sensitivity in cancer. Nature 459: 1085-1090, 2009.

17. $\mathrm{Hu} \mathrm{BS}, \mathrm{Hu} \mathrm{H}, \mathrm{Zhu} \mathrm{CY}, \mathrm{Gu}$ YL and Li JP: Overexpression of GOLPH3 is associated with poor clinical outcome in gastric cancer. Tumour Biol 34: 515-520, 2013.

18. Peng J, Fang Y, Tao Y, Li K, Su T, Nong Y, Xie F and Lai M: Mechanisms of GOLPH3 associated with the progression of gastric cancer: A preliminary study. PLoS One 9: e107362, 2014.

19. Dippold HC, Ng MM, Farber-Katz SE, Lee SK, Kerr ML, Peterman MC, Sim R, Wiharto PA, Galbraith KA,Madhavarapu S, et al: GOLPH3 bridges phosphatidylinositol-4-phosphate and actomyosin to stretch and shape the Golgi to promote budding. Cell 139: 337-351, 2009.

20. Yang Q and Guan KL: Expanding mTOR signaling. Cell Res 17: 666-681, 2007.

21. Ueda T, Volinia S, Okumura H, Shimizu M, Taccioli C, Rossi S, Alder H, Liu CG, Oue N, Yasui W, et al: Relation between microRNA expression and progression and prognosis of gastric cancer: A microRNA expression analysis. Lancet Oncol 11: 136-146, 2010.

22. Zhu C, Ren C, Han J, Ding Y, Du J, Dai N, Dai J, Ma H, Hu Z, Shen $\mathrm{H}$, et al: A five-microRNA panel in plasma was identified as potential biomarker for early detection of gastric cancer. Br J Cancer 110: 2291-2299, 2014.

23. Han C, Zhou Y, An Q, Li F, Li D, Zhang X, Yu Z, Zheng L, Duan Z and Kan Q: MicroRNA-1 (miR-1) inhibits gastric cancer cell proliferation and migration by targeting MET. Tumour Biol 36: 6715-6723, 2015.

24. Riquelme I, Tapia O, Leal P, Sandoval A, Varga MG, Letelier P, Buchegger K, Bizama C, Espinoza JA, Peek RM, et al: miR-101-2, miR-125b-2 and miR-451a act as potential tumor suppressors in gastric cancer through regulation of the PI3K/AKT/mTOR pathway. Cell Oncol 39: 23-33, 2016.

25. Ding L, Xu Y, Zhang W, Deng Y, Si M, Du Y, Yao H, Liu X, Ke Y, Si J, et al: MiR-375 frequently downregulated in gastric cancer inhibits cell proliferation by targeting JAK2. Cell Res 20: 784-793, 2010. 
26. Zhang X, Yan Z, Zhang J, Gong L, Li W, Cui J, Liu Y, Gao Z, Li J, Shen L, et al: Combination of hsa-miR-375 and hsa-miR-142-5p as a predictor for recurrence risk in gastric cancer patients following surgical resection. Ann Oncol 22: 2257-2266, 2011

27. Chen LG, Wang HJ, Yao HB, Guan TP, Wu F, He XJ, Ma YY, Tao HQ and Ye ZY: GP73 is down-regulated in gastric cancer and associated with tumor differentiation. World J Surg Oncol 11: $132,2013$.

28. Sechi S, Frappaolo A, Belloni G, Colotti G and Giansanti MG: The multiple cellular functions of the oncoprotein Golgi phosphoprotein 3. Oncotarget 6: 3493-3506, 2015.
29. Farber-Katz SE, Dippold HC, Buschman MD, Peterman MC, Xing M, Noakes CJ, Tat J, Ng MM, Rahajeng J, Cowan DM, et al: DNA damage triggers Golgi dispersal via DNA-PK and GOLPH3. Cell 156: 413-427, 2014.

30. Hohenberger P and Gretschel S: Gastric cancer. Lancet 362: 305-315, 2003.

31. Murayama T, Inokuchi M, Takagi Y, Yamada H, Kojima K, Kumagai J, Kawano T and Sugihara K: Relation between outcomes and localisation of p-mTOR expression in gastric cancer. $\mathrm{Br} \mathrm{J}$ Cancer 100: 782-788, 2009. 